\title{
From Diagnosis to Remission: A Five-Year Case Study of a Female Collegiate Athlete's Battle with Leukemia
}

\author{
Kayla N. Middle ton ${ }^{1}$, Nicole M. Glasgow ${ }^{1}$, Holly E. King ${ }^{1}$, Mary E. Jacobs ${ }^{2}$, Andrew T. Doyle ${ }^{2}$, \\ Adam J. Thompson",
}

${ }^{1}$ Athletic Training Education, Indiana Wesleyan University, Marion, 46953, USA

${ }^{2}$ Athletic Training Services, Indiana Wesley an University, Marion, 46953, USA

\begin{abstract}
This case follows a female collegiate basketball player who was diagnosed with Acute Lymphocytic Leukemia (ALL). Th is five-year study explores the unique aspects of this subject's case by examin ing each characteristic of her journey from init ial diagnosis as an undergraduate freshman through treatment and into remission by her senior year. Participation in collegiate athletics was identified for its positive influence in serving as a modality to overcome this oncologic condition that affected the mental, physical and emotional state of the athlete. The first part of this study examines the subject's battle with cancer uniquely occurring overher five years of undergraduate study. Follo wing the case report, the physical effects of cancer on the body are discussed as well as the benefits of exerc ise for cancer patients. To conclude the discussion, the psychological implications of cancer and tools used to combat the negative consequences on the mind are reviewed. This study concludes that physical activity in conjunction with the integration of support from her collegiate basketball team contributed to the positive outcomes of the subject's psychological and physical well-being.
\end{abstract}

Keywords Acute Lymphocytic Leukemia (ALL), Osteonecrosis

\section{Introduction}

Acute lymphocytic leukemia is characterized by immature ly mphocytes proliferating into the bone marrow. ${ }^{[1]}$ ALL is the least common type of leukemia, but it is most common in children. Generally, ALL occurs before the age of fourteen with peak incidence in two to nine year olds. ${ }^{[1]}$ This is a presentation of a female collegiate basketball player who was not diagnosed with ALL until she was eighteen years of age; the first of many items which classify her as an atypical leukemia patient and survivor. This five-year study explores the unique aspects of the subject's case by examining each characteristic of her journey from initial diagnosis as an undergraduate freshman through treatment and into remission by her senior year.

Not only did this athlete work to overcome cancer, but she fought to return to play after suffering from bilateral osteonecrosis in her knees due to chemotherapy treatments. Research has shown that although ALL survival rates have increased with the use of multiple chemotherapeutic agents, many of these treatments can cause complications affecting the musculoskeletal and neuro muscular systems. ${ }^{[2]}$ Statistics show that $38 \%$ of children who get treated for ALL develop

* Corresponding author:

adam.thompson@indwes.edu (Adam J. Thompson)

Published online at http//journal.sapub.org/ijtt

Copyright (C) 2012 Scientific \& Academic Publishing. All Rights Reserved osteonecrosis. Furthermore, the maturing bones of adolescents may be more susceptible to develop osteonecrosis in comparison to younger children because of epiphyseal closure resulting in marrow ischemia and reduced blood flow.

Leuke mia has many physical effects on the body and tends to put a halt on life. ${ }^{[1,3]}$ Because of this, the question for the case study became 'How does participating in a sport positively or negatively affect an individual with ALL?' To answer this question, simply looking at the benefits of exercise will not suffice. Instead, one should look at the situation holistically by a lso taking into account the psychosocial facet of being a part of a team ${ }^{[8]}$.

\section{Case Report}

A high school star basketball player was transitioning from her senior year of high school into intercollegiate competition. During the week of August 19, 2005 this female athlete had her wisdom teeth removed. Following the surgery, she developed a high fever and was taken to the hospital. After two blood tests were administered to determine the cause of the high fever, she was diagnosed with acute lymphocytic leukemia; a cancer where blood cells never fully mature. In her specific case, $97 \%$ of those cells were cancerous, and the doctors estimated that she had a $70 \%$ chance of survival. Immediately following the diagnosis, she began chemotherapy treatments through a port once a week and daily took oral medications. All basketball activities 
were $d$ iscontinued.

Several times during initial treatment, the athlete's blood counts were too low for the physicians to perform the chemotherapy. Because of this, treatments were sometimes postponed. The patient was forced to avoid environments of dense population because of her increased chance of contracting illness. Therefore, she was not allowed to live in community environments on campus during the first semester of college. Fortunately, she was still able to take classes and made it to team practices and games as her physicians permitted. One month into her chemotherapy treatments, this athlete suffered a stroke believed to be caused by one of her medications called Doxorubicin. This stroke caused fatigue and a decline in the peripheral vision of her right eye; this conditions eventually resolved. Three months after her stroke, the athlete became very sick and was eventually diagnosed with Histoplasmosis, a fungal infection.

In order to get overcome this fungal infection, she had to stay in the hospital for two weeks. During the second semester of her freshman year, she was allowed to live on campus, but continued to have frequent chemotherapy treatments. At this time, she started progressing through the third and fourth stages of chemotherapy and was allowed to start light conditioning and practicing with the women's basketball team. As she began doing so, she experienced knee pain but claimed that it was tolerable. She managed her knee pain with Cryotherapy treatments. During the summer before her sophomore year, she concentrated heavily on gaining strength and endurance in order to play basketball in the fall.

In the fall of 2006, this athlete was cleared to return as a full time academic student and intercollegiate basketball player. She began practicing with the women's basketball team but was only required to participate in low intensity drills. Although she began to practice with the team, her practice schedule varied depending on her physical status. Her blood counts were still closely monitored, and whenever the hemoglobin count was too low, her physical activity would be temporarily postponed.

In November of 2006 she began working back into full scale practices and developed substantial pain and inflammation on the lateral aspect of both of her knees. Upon evaluation she was diagnosed with iliotibial band tendonitis in her right knee and synovitis of her left knee by the supervising licensed athletic trainer. Conservative treatment with ultrasound and ice along with stretching followed. The condition and pain did not resolve or respond to the treatments. Pain progressively worsened and began to move throughout her knees. Due to the knee pain and resulting limitations, she had bilateral radiographs on January 22, 2007. The radiographs identified lateral femoral avascular necrosis of her left knee which was believed to be caused by one of her medications called Dexamethasone. Seven days later an MRI showed that she had severe osteonecrosis in both knees, but the left knee demonstrated significantly worse pathology. The athlete was instructed to avoid high impact activities, and therefore, basketball was not an option. Although her basketball career was put to a halt once again, she was still a part of the team. She attended all practices and games and claimed that she was the "team captain of the bench." No further participation was attempted during the 2006-2007 basketball season.

In May 2007, this athlete underwent arthroscopic surgery on her left knee. During this operation, the surgeon discovered that the knee condition was far worse than expected. A portion of the lateral knee had eroded due to her condition, and pieces of chondral bone and articular cartilage needed to be debrided and removed.

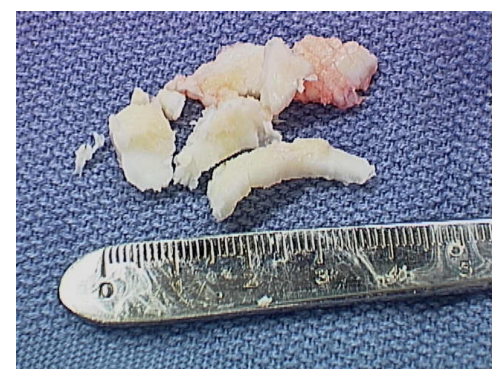

Figure 1. A sampling of bone and cartilage debris that was removed from the athlete's knee during arthroscopic surgery in May 2007

The athlete was then placed on a donor list for a bone/cartilage graft due to the loss of these critical tissues. In September 2007, she underwent an osteochondral allograft transplant and micro-fracture surgery. A lthough she would not be able to play basketball her junior year because of the surgery, she still had the goal of eventually playing college basketball. Her role on the team th is year was as the student assistant coach and statistician. On the positive side, she was able to stop her oral chemotherapy treatments in December 2007. Three months later, her cancer was in remission.

Eight months after the surgery, her orthopedic surgeon cleared her to slowly start running. By the beginning of the school year, she could run every other day. On September $23^{\text {rd }}, 2008$, a little over a year past the surgery, her surgeon removed all activity limitations. She was then able to play the 2008-2009 and 2009-2010 seasons without restrictions. Even while she was playing her last two seasons, she still underwent cancer screenings every three months. Her initial diagnosis in late summer 2005 significantly compromised her colleg iate basketball career. Due to the cancer, her participation was sporadic but possible and upon her graduation in April of 2010 her acute ly mphocytic leukemia was still in remission.

\section{Discussion}

Leukemia is defined as a group of malignant disorders that affect the blood and the blood forming tissues. ${ }^{[1]}$ The four main subcategories of leukemia include acute myelocytic (AML), acute lymphocytic (ALL), chronic myelocytic (CML), and chronic ly mphocytic (CLL). Classification of leukemia type is based on the speed of onset as well as the type of white blood cells that are affected. Though leukemia is often thought of as a childhood cancer, it occurs within all 
age populations with approximately 34,810 new cases being diagnosed every year. ${ }^{[1]}$ Acute lymphocytic leukemia is diagnosed through blood tests and bone marrow exams. ${ }^{[1]}$ Chemotherapy is the most used treatment for ALL, often accompanied by blood transfusions, antibiotics, antivirals, and bone marrow transplants. Since the 1970s the five year survival rate of ALL has increased in adults from $38 \%$ to $65 \%$, and from $53 \%$ to $85 \%$ in children. ${ }^{[4]}$ These statistics match up with the survival chance given to the subject of this study by her physician (70\%).

Because there has been such an increase in the survival ratings of ALL and doctors have found effective protocols to combat the cancer, research is now being done to determine the long term effects of the treatments. ${ }^{[2]}$ Specifically, Dexamethasone and Prednisone are known to cause osteonecros is in those with ALL and can develop two months to five years after diagnosis. The anterior lateral region of the femoral head, the distal femur, and the proximal tibia are the most frequently involved sites for osteonecrosis of the knee. In this particular case, the distal femur was the affected area.

As one can see from this case report, leukemia affects the human body in many ways. In fact, leukemia can lead to feelings of fatigue, atrophy of muscle tissue, and the breakdown of other connective tissues such as ligaments and tendons. ${ }^{[3]}$ Diagnostically a person can present with low counts of red blood cells, hemoglobin, and platelet; as well as a low white blood cell count, high $\mathrm{LDH}$, and hypercellular bone marrow with lymphoblasts. ${ }^{[3]}$ In addition to what was previously mentioned, a person with ALL may experience fever, pallor, weakness, bone, joint, and abdominal pain, infections, weight loss, hepatosplenomegaly, headache, or cranial nerve dysfunction ${ }^{[1]}$.

Current research suggests that exercising and engaging in physical activity with cancer benefits one's physical body. ${ }^{[5]}$ According to the American College of Sports Medicine, cancer patients and survivors need to have activity levels that are as close to normal as possible. The 2008 Federal Physical Activity Gu idelines for Americans suggests approximately 150 minutes of moderate aerobic exercise each week, which also applies to those battling cancer ${ }^{[6]}$.

The subject of this case continued to exercise as much as she was able throughout her battle with ALL and into remission. Throughout the process, she participated in basketball as able as well as other modes of exercise including bicycle, elliptical, and swimming. Her main limitation during treatment was fatigue, and as she developed osteonecrosis of her knees, she was mostly limited by pain. These limitations primarily affected the type of exercise, but also affected the frequency. In fact, exercise may have helped her to overcome the disease as she was engaged in regular exercise throughout the treatment process. Physical activity during cancer diagnosis, treatment, and remission can help to decrease the effects of fatigue, maintain physical fitness levels, reduce the risk of cardiovascular complications, increase life span by having a positive impact on the oncologic condition, and have a positive influence on the psychological component of recovery. ${ }^{[3,7-9]}$ Cancer-related fatigue is a term defined by the National Co mprehensive Cancer Network as a "persistent and subjective sense of tiredness related to cancer or cancer treatment that interferes with usual functioning," and reportedly affects $70-100 \%$ of all cancer patients. ${ }^{[1]}$ Some believe that because leukemia patients complain of fatigue, they should rest in order to maintain their strength. However, too often this can lead to a cycle of de-conditioning which involves a decline in psychological, emotional, and physical function. ${ }^{[3]}$ A study from the University of North Carolina at Chapel Hill found that leukemia patients who took part in a three week individualized exercise program had significantly reduced levels of perceived fatigue and depression than those who did not exerc is $\mathrm{e}^{[9]}$.

The most basic physical effects of exercise are improvements in cardiovascular health and decreased body weight, both of which can reduce one's risk of heart attack or stroke. ${ }^{[10]}$ Cancer patients are not immune from these conditions and in fact may be more susceptible to them. ${ }^{[11]}$ The general decreased fitness levels and obesity found in cancer survivors today may be primarily a result of leading a sedentary lifestyle as opposed to the initial assumption of a decreased exercise capacity. ${ }^{[12]}$ In the case of this patient, the athlete gained weight throughout the cancer treatment process. At the end of the treatment the athletic trainer working with the patient, was able to help her address this issue through a strict exercise and diet plan. Through this program, she was able to lose 20 pounds and return to her healthy weight. It was through the instruction of her athlet ic trainer that she was able to be successful in this endeavor. One of the benefits of being a part of the college basketball team was her access to an athletic trainer who had the knowledge base and resources to help her return to a healthier weight without further complications and injuries. Her participation in exercise during remission has been vital to her overall health and offers an improved life for many years to come. In addition to these basic physical benefits of exercise in cancer patients, recent research has shown a correlation between exercise in cancer patients and the defeat of the oncologic condition as well as the prevention of the disease returning. ${ }^{[7,13]}$ In two different research studies by Harvard University, it was discovered that implementing a regular walking program in patients with cancer, or currently in remission, for three to six hours per week, greatly increases their chances of survival. ${ }^{[7]}$ Additionally, it has been established that the better the physical condition of a person when diagnosed with cancer, the more likely they are to survive the disease. ${ }^{[13]}$ The subject may have greatly benefited from previously being involved in athletics in that it may have helped in her fight against this oncologic condition. Physical fitness is important for every individual, which the subject of this case study found the same to be true.

As demonstrated in the subject's case, there is evidence to suggest that physical activity may improve the psychosocial aspects of surviving cancer as well. ${ }^{[8]}$ Depression and anxiety-related issues have been shown to decrease among cancer patients who take part in exercise during treatment. ${ }^{8}$ Cancer treatment and exercise should be united together as 
exercise may help cancer patients to feel rejuvenated, and overall, help to treat cancer long-term. Exercise is clearly beneficial to cancer patients, which is one of two major components to being part of a basketball team along with the psychos ocial benefits.

Furthermore, research suggests that more important than having optimistic expectations about one's cancer prognos is is the avoidance of pessimistic expectations. ${ }^{[14]}$ Involvement in basketball was able to give the patient another outlet of activity. She had other things to focus on besides just the cancer, while at the same time the support of the team reportedly benefited her optimistic expectations.

When a person is battling cancer, one must consider the deep psychological impact that results as patients face new fears such as treatment, sickness, an unknown future, and even potential death. The cancer patient may progress through Kubler-Ross' Stages of Death beginning with denial and progressing through anger, bargaining, depression, and finally toward acceptance.

It is suggested that dealing with sickness and injury may generally be even more difficult for athletes compared to the general population. ${ }^{[15]}$ This is due to being forced not to participate in their sport, and the concern and anxiety that often arise with how long it will take them to heal. ${ }^{[15]}$ So me common psychological effects that are seen in athletes who must sit out of their sport due to injury are anger, depression, anxiety, tension, fear, and lower self-esteem. ${ }^{[15]}$ Because of these possible changes, an individual's personality may be compromised when faced with a debilitating disease. ${ }^{14}$ Injured athletes have to learn to appropriately cope mentally, emotionally, and behaviorally. Because of these things, social support is very important for an athlete who is going through rehabilitation ${ }^{[16]}$.

In the situation of this case study, the athlete was going through cancer treatment and eventually reconditioning so she could return to playing basketball. Social support can be defined as "an exchange of resources between two individuals perceived by the provider or the recipient to be intended to enhance the well-being of the recipient." ${ }^{[16]}$ Social support is a way to make up for something the athlete has lost due to injury and lessen some of the negative impact. ${ }^{[17]}$ Yang, et al. suggests that there are eight different kinds of support: listening support, emotional support, emotional support challenge, reality confirmation, task challenge, task appreciation, tangible support, and personal ass istance. The important thing is to carefully assess the athlete's needs so that the proper kind of support can be given at the appropriate time ${ }^{[18]}$.

There are several different individuals and groups of people who can be a part of an athlete's social support network. Coaches, tea mmates, friends, family members, athlet ic trainers, and other medical practitioners all have special opportunities to encourage the injured athlete. In the case of the athlete of this discussion, her coach and teammates were a very strong source of support for her. It is very important that while an athlete is out of play, they still need to be involved with their team, and feel that they still have a place on the team. ${ }^{16}$ While this athlete was unable to take part in full practices or compete for a few years, her coach was very active in giving her the roles of team statistician and mentor to her teammates. The athlete states that she never had to wonder if she had a place on the team. This is even more important in college athletics since the students are often far from home, and therefore need other sources of support when they are not around their families ${ }^{[15]}$.

Positive social support, as demonstrated by the subject's parents, teammates, and others around her, created an optimal environment to foster positive emotional and psychosocial adjustment during her fight with leukemia.

Being a part of a team while she battled ALL was very important in this athlete's case. One way it benefitted her was that it gave her goals to strive for. Goals are extremely important because they give people a sense of identity and accomplishment. ${ }^{[19]}$ It also gave her an opportunity to stay active and be part of a tightly knit source of support. It could even be argued that being on a basketball team, which is a relatively small group of people, was even more beneficial than other potential groups of support sources that are larger. In a study done by Carron and Spink ${ }^{[20]}$, they found that smaller groups had greater perceptions of cohesion than larger groups, meaning they are mo re likely to stay together and be united in achieving its goals. The amount of time the group spent together was also found to be very indicative of their perceptions of cohesion. In the case of a basketball team, they spend hours each day together at practices and games alone. Because motivation and team cohesion are the two primary reasons people play sports, this type of social cohesion would be very valuable to this athlete. ${ }^{[21]}$ In addition to her team, this athlete also was able to form a relationship with the team's athletic trainer. In a recent study, researchers found that "athletic trainers may be among the most effective sources of high quality support for injured athletes."[15] While the athletic trainer did not, obviously, take part in her cancer treatments, he had a very large role in managing and treating her knees, as well as giving her a plan for weight loss and reconditioning. This reconditioning was done during practice so that she could still interact with her team.

\section{Conclusions}

The case of this women's basketball player and her five-year battle with ALL is an excellent example of the benefits of being part of a sport's team while battling cancer. Her involvement in athletics gave her an alternative treatment, as she was able to participate with the team in varying degrees throughout her treatment and into remission, so that she was able to reap the benefits of exercise. In addition, upon interviewing the athlete, it is clear that the psychosocial aspect of being part of a team provid ing social support had a positive effect on her recovery. While these things proved beneficial for the athlete of this particular case, they may not have the same effect on other athletes. Therefore, it would be advantageous for more research to be done in this area with 
other cases.

Additional benefits to the athlete were access to athletic trainers who were able to assist her at various points of treatment throughout the recovery process. The athletic trainers played a vital role in assisting her return to a healthy weight, rehabilitation of osteonecrosis, as well as monitoring her overall well-being throughout her years as a collegiate athlete. One significant implication of this case is the importance for the caregivers to be aware of the increased possibility of osteonecrosis in athletes receiving cancer treatment and taking Dexamethasone for extended periods of time. Clinicians need to be proactive in recognizing and diagnosing the condition in order to minimize the extent of damage.

Further research on osteonecrosis and the effects of exercise on the development of this condition would be beneficial as well. Another important implication of this case is to have the athlete involved with the team as much as possible, even if that means doing separate workouts on the sidelines during practice. This was found to increase the positive effects of the social support of the team.

Furthermore, it is beneficial for the coach to be informed of the importance of including the athlete in tea mactivities in order to get the most benefit from the team's social support. In addition, as athletic trainers it is imperative to remember that even though the athlete may be suffering from fatigue it is important to encourage exercise in order to battle the fatigue. It can be beneficial if the athletic trainer is able to give a wide variety of activities for the athlete to be involved in, which can foster more interest for the athlete. Further research should be done to establish the balance between the use of rest and exercise to battle cancer-related fatigue.

Original version of this temp late was provided by courtesy of SAP Productions.

\section{ACKNOWLEDGEMENTS}

We would like to acknowledge the physicians involved in the care of this patient for their willingness to provide extensive information regarding the treatment and diagnostic methods used in the care for this patient. We would also like to acknowledge the patient for their cooperation in secure information and reports to accurately document their medical history and journey towards beating cancer.

\section{REFERENCES}

[1] Lewis, S., Heitkemper, M., Dirkson, S., O’Brien, P., \& Bucher, L. Medical-Surgical Nursing. St. Louis, MO: Mosby Elsevier; 2007.

[2] Marchese, V., Connolly, B., Able, C., Booten, A., Bowen, P., Porter, B., Rai, S., Han cock, M., Pui, C., Howard, S., Neel, M., $\&$ Kaste, S. Relationships among severity of osteonecrosis, pain, range of motion, and functional mobility in children, adolescents, and young adults with acute lymphoblastic leukemia. Physical Therapy. 2008;88(3):341-350.

[3] Douglas, E. Exercise in cancer patients. Physical Therapy Reviews, 2005;10:71-88.

[4] Huether, S. \& McCance, K. Understanding Pathophysiology. St. Louis, MO: Mosby Elsevier; 2008.

[5] Watson, T., \& Mock, V. Exercise as an intervention for cancer-related fatigue. Physical Therapy. 2004;84:736-743.

[6] American college of sports medicine: New guidelines strongly recommend exercise for cancer patients, survivors. Available at http://www.acsm.org/about-acsm/media-room/a csm-in-the-news/2011/08/01/new-guidelines-strongly-recom mend-exercise-for-cancer-patients-survivors. Retrieved M arc h 2012.

[7] Klebes, T. Giving Cancer its walking papers. Harvard Health Letter. 2006;7. Available at http://www.health.harvard.edu. Retrieved January 2012.

[8] Schwartz, A. L. Physical activity after a cancer diagnosis: psychosocial outcomes. Cancer Investigation. 2004;22(1).

[9] Science Daily. Exercise benefits leukemia patients. 2009. Available at http://www.sciencedaily.com/releases/2009/08/ 090803083635. Retrieved January 2012.

[10] Yung, L. M., Laher, I., Yao, X., Chen, Z. Y., Huang, Y., \& Leung, F. P. Exercise, vascular wall and cardiovascular diseases. Sports Medicine. 2009;39 (1): 45-63.

[11] Brussel, M.V., Takken, T., Van Der Net, J., Engelbert, R.H.H., Bierings, M., Schoenmakers, M.A.G.C., \& Helders, P.J.M. Physical function and fitness in long-term survivors of childhood leukemia. Pediatric Rehabilitation. 2006;9:267-27 4.

[12] Bar, G., Black, P.C., \& Gutjahr, P. Recovery kinetics of heart rate and oxygen uptake in long-term survivors of acute leukemia in childhood. Eur J Pediatr. 2007;166:1135-1142.

[13] Adkins, B.W. Maximizing exercise in breast cancer survivors. Clinical Journal of Oncology Nursing. 2009;13:695-700.

[14] Pinquart, M., Frohlich, C., \& Silbereisen, R. K. Optimism, pessimism, and change of psy chological well-being in cancer patients. Psychology, Health, \& Medicine. 2007;12(4).

[15] Yang, J., Peek-Asa, C., Lowe, J. B., Heiden, E., \& Foster, D. T. Social Support Patterns of Collegiate Athletes Before and After Injury. Journal Of Athletic Training. 2010;45(4):372-3 79.

[16] Corbillon, F., Crossman, J., \& Jamieson, J. Injured Athletes' Perceptions of the Social Support Provided by Their Coaches and Teammates During Rehabilitation. Journal Of Sport Behavior. 2008;31(2):93-107.

[17] Ford, I. W., \& Gordon, S. Coping with sport injury: Resource lost and the role of social support. Journal Of Personal \& Interpersonal Loss. 1999;4(3):243.

[18] Mitchell, I. Social support and psychological responses in sport-injury rehabilitation. Sport \& Exercise Psychology Review. 2011;7(2):30-44.

[19] Schroevers, M., Kraaij, V., \& Garnefski, N. How do cancer patients manage unattainable personal goals and regulate their emotions? British Journal of Health Psychology. 2008;13. 
[20] Carron, A. V., \& Spink, K. S. The group size-cohesion relationship in minimal groups. Small Group Research. 1995;26(1):86.
[21] Halbrook, M., Blom, L. C., Hurley, K., Bell, R. J., \& Holden, J. E. Relationships among Motivation, Gender, and Cohesion in a Sample of Collegiate Athletes. Journal OfSport Behavior. 2012;35(1):61-77. 